\title{
On generalizations of quasi-prime ideals of an ordered left almost semigroups
}

\author{
Pairote Yiarayong ${ }^{1}$
}

Received: 9 May 2019 / Accepted: 2 January 2021 / Published online: 31 January 2021

(c) The Author(s) 2021

\begin{abstract}
The purposes of this paper are to introduce generalizations of quasi-prime ideals to the context of $\phi$-quasi-prime ideals. Let $\phi: \mathcal{I}(S) \rightarrow \mathcal{I}(S) \cup\{\emptyset\}$ be a function where $\mathcal{I}(S)$ is the set of all left ideals of an ordered $\mathcal{L} \mathcal{A}$-semigroup $S$. A proper left ideal $A$ of an ordered $\mathcal{L} \mathcal{A}$-semigroup $S$ is called a $\phi$-quasi-prime ideal, if for each $a, b \in S$ with $a b \in A-\phi(A)$, then $a \in A$ or $b \in A$. Some characterizations of quasi-prime and $\phi$-quasi-prime ideals are obtained. Moreover, we investigate relationships between weakly quasi-prime, almost quasiprime, $\omega$-quasi-prime, $m$-quasi-prime and $\phi$-quasi-prime ideals of ordered $\mathcal{L} \mathcal{A}$-semigroups. Finally, we obtain necessary and sufficient conditions of $\phi$-quasi-prime ideal in order to be a quasi-prime ideal.
\end{abstract}

Keyword ordered $\mathcal{L} \mathcal{A}$-semigroup, quasi-prime ideal, $\phi$-quasi-prime ideal, $\omega$-quasi-prime, $\phi$-zero.

Mathematics Subject Classification 20M10 • 16Y99.

\section{Introduction}

In 2010, Shah et al. [35] studied ideals, $M$-systems, $N$-systems and $I$-systems of ordered $\mathcal{L} \mathcal{A}$-semigroups and provided that if $A$ is a left ideal of an ordered $\mathcal{L} \mathcal{A}$-semigroup with left identity, then $A$ is quasi-prime if and only if $S-A$ is an $M$-system; $A$ is quasi-semiprime if and only if $S-A$ is an $N$-system and $A$ is quasi-irreducible if and only if $S-A$ is an $I$ system. Nowadays many scholars have studied different aspects of ordered $\mathcal{L} \mathcal{A}$-semigroups see $[4,7,14,33,44,47-49]$. In 2012, Faisal et al. [13] introduced the notion of fuzzy ordered $\Gamma$ - $\mathcal{L} \mathcal{A}$-semigroups and studied $(2,2)$-regular ordered $\Gamma$ - $\mathcal{L} \mathcal{A}^{* *}$-semigroup in terms of fuzzy $\Gamma$-left ideals, fuzzy $\Gamma$-right ideals, fuzzy $\Gamma$-two-sided ideals, fuzzy $\Gamma$-generalized bi-ideals, fuzzy $\Gamma$-bi-ideals, fuzzy $\Gamma$-interior ideals and fuzzy $\Gamma$-(1;2)-ideals. They proved that the set of all fuzzy $\Gamma$-two-sided ideals of a $(2,2)$-regular ordered $\Gamma$ - $\mathcal{L} \mathcal{A}^{* *}$-semigroup $S$ forms a

Pairote Yiarayong

pairote0027@hotmail.com

1 Department of Mathematics, Faculty of Science and Technology, Pibulsongkram Rajabhat University, Phitsanulok 65000, Thailand 
semilattice structure with identity $S$. In 2013, Khan et al. [18] characterized an intra-regular ordered $\mathcal{L} \mathcal{A}$-semigroup in terms of interval valued fuzzy left (right, two-sided) ideals. In 2014, Yousafzai et al. [46] introduced the notion of fully regular ( $V$-regular) class of an ordered $\mathcal{L} \mathcal{A}$-semigroup and characterized fully regular $(V$-regular) class of an ordered $\mathcal{L} \mathcal{A}$ semigroup in terms of fuzzy (left, right, two-sided, interior, bi-, generalized bi- and quasi) ideals. In 2015, Khan et al. [24] defined (0,2)-ideals and (1,2)-ideals of an ordered $\mathcal{L} \mathcal{A}$ semigroups and proved that the ordered $\mathcal{L} \mathcal{A}$-semigroup $S$ is 0 -(0,2)-bisimple if and only if $S$ is right 0-simple. In 2016, Yousafzai et al. [51] introduced the notion of $\left(\in_{\gamma}, \in_{\gamma} \vee q_{\delta}\right)$ fuzzy (left, right, bi-) ideals of an ordered $\mathcal{L} \mathcal{A}$-semigroup and characterized intra-regular ordered $\mathcal{L} \mathcal{A}$-semigroups in terms of these generalized fuzzy ideals. In 2017, Yiarayong [42] have also studied prime, semiprime, quasi-prime and quasi-semiprime ideals of ordered $\mathcal{L} \mathcal{A}$ semigroups. In 2018, Amjid et al. [6] introduced the notion of smallest one-sided ideals in an $\mathcal{L} \mathcal{A}$-semigroup.

In 2004, Stevanovic and Proti [37] introduced the notion of a 3-potent element of an $\mathcal{L} \mathcal{A}$-semigroup and of AG-3-band. They studied several properties of AG-3-bands and $A G$-bands. Nowadays many scholars have studied different aspects of $\mathcal{L} \mathcal{A}$-semigroups and $\mathcal{L} \mathcal{A}$-semihypergroups see [5,10,16,19,22,23,26,29,32,39,40]. In [27,28] Mushtaq and Khan (2006-2007) initiated the study of AG-bands and $\mathrm{AG}^{*}$-groupoids. They proved that an ideal $A$ of an AG-band is prime iff if ideal $(S)$ is totally ordered; it is prime iff it is strongly irreducible. In 2012, Khan and Anis [17] proved that $S / \gamma$ is a maximal separative semilattice homomorphic image of an $\mathcal{L} \mathcal{A}$-semigroup $S$. In 2013, Shah and Rehman [17] studied several properties of locally associative $\Gamma$ - $\mathcal{L} \mathcal{A}$-semigroups. They proved that for a locally associative $\Gamma$ - $\mathcal{L} \mathcal{A}$-semigroup $S$ with a left identity, $S / \rho$ is a maximal weakly separative homomorphic image of $S$, where $\rho$ is a relation on $S$ defined by: $a \rho b$ if and only if $a \gamma b^{n}=b^{n+1}$ and $b \gamma a^{n}=a^{n+1}$ for some positive integer $n$ and for all $\gamma \in \Gamma$, where $a, b \in S$. In 2014, Abdullah et al. [3] introduced the concept of interval-valued $(\in, \in \vee q)$-fuzzy ideals, interval-valued $(\in, \in \vee q)$-fuzzy bi-ideals and interval-valued $(\in, \in \vee q)$-fuzzy quasi-ideals of an $\mathcal{L} \mathcal{A}$-semigroup. Nowadays many scholars have studied different aspects of fuzzy subsets on $\mathcal{L} \mathcal{A}$-semigroups and $\Gamma$ - $\mathcal{L} \mathcal{A}$-semigroups see $[9,11,12,15,20,30,31,36,38,41,45,50]$. In 2015, Abbasi and Basar [1] studied quasi-ideals and bi-ideals of locally associative $\Gamma$ $\mathcal{L} \mathcal{A}$-semigroups, $(m, n)$ simple $\Gamma$ - $\mathcal{L} \mathcal{A}$-semigroups, minimal bi- $\Gamma$-ideal, semiprime $\Gamma$-ideal and quasi-regular $\Gamma$ - $\mathcal{L} \mathcal{A}$-semigroups. They proved that the product of two $(m, n)-\Gamma$-ideals of a locally associative $\Gamma$ - $\mathcal{L} \mathcal{A}$-semigroup $S$ with left identity is an $(m, n)-\Gamma$-ideal of $S$. In 2016, Khan et al. [25] have also studied $(m, n)$-ideals and 0 -minimal $(m, n)$-ideals of $\mathcal{L} \mathcal{A}$ semigroups and proved that $A$ is a $(0,2)$-ideal of $S$ if and only if $A$ is a left ideal of some left ideal of $S$. In 2017, Khan et al. [21] defined $(\alpha, \beta)$-fuzzy bi-ideals, $(\alpha, \beta)$-fuzzy interior ideals, $(\bar{\beta}, \bar{\alpha})$-fuzzy bi-ideals and $(\bar{\beta}, \bar{\alpha})$-fuzzy interior ideals in $\mathcal{L} \mathcal{A}$-semigroups. In 2019 , Younas and Mushtaq [43] proved that the set of idempotent elements in a left permutable inverse $\mathcal{L} \mathcal{A}$-semigroup is an order ideal. In 2020, Abbasi et al. [2] introduced the notion of soft interior-hyperideals in $\mathcal{L} \mathcal{A}$-semihypergroups. They studied several properties of soft interior-hyperideals of LA-semihypergroups.

Motivated and inspired by the above works, the aim of this paper is to extend the concept of quasi-prime ideals in multiplicative hyperrings given by Yiarayong [42] to the context of $\phi$-quasi-prime ideals. Let $\phi: \mathcal{I}(S) \rightarrow \mathcal{I}(S) \cup\{\varnothing\}$ be a function where $\mathcal{I}(S)$ is the set of all left ideals of $M$. A proper left ideal $A$ of an ordered $\mathcal{L} \mathcal{A}$-semigroup $S$ is called a $\phi$-quasi-prime ideal, if for each $a, b \in S$ with $a b \subseteq A-\phi(A)$, then $a \in A$ or $b \in A$. Some characterizations of quasi-prime and $\phi$-quasi-prime ideals are obtained. Moreover, we investigate relationships between weakly quasi-prime, almost quasi-prime, $\omega$-quasi-prime, $m$-quasi-prime and $\phi$ - 
quasi-prime ideals of ordered $\mathcal{L} \mathcal{A}$-semigroups. Finally, we obtain necessary and sufficient conditions of $\phi$-quasi-prime ideal in order to be a $\phi$-quasi-prime ideal.

\section{$2 \phi$-quasi-prime ideals of ordered $\mathcal{L} \mathcal{A}$-semigroups}

In this section, we give some basic properties of $\phi$-quasi-prime ideals and investigate $\phi$ quasi-prime ideals in several classes of ordered $\mathcal{L} \mathcal{A}$-semigroups and give its characterizations corresponding to $\phi$-quasi-prime ideals in ordered $\mathcal{L} \mathcal{A}$-semigroups.

For the sake of completeness, we state some definitions in the same fashion as found in [8] which are used throughout this paper.

Definition 1 Let $S$ be an ordered $\mathcal{L} \mathcal{A}$-semigroup and let $\phi: \mathcal{I}(S) \rightarrow \mathcal{I}(S) \cup\{\emptyset\}$ be a function where $\mathcal{I}(S)$ be a set of all left ideals of $S$. A proper left ideal $A$ of $S$ is called a $\phi$-quasi-prime ideal if for each $a, b \in S$ with $a b \in A-\phi(A)$, then $a \in A$ or $b \in A$.

We now present the following example satisfying above definition.

Example 1 Let $S=\{a, b, c\}$ be an ordered $\mathcal{L} \mathcal{A}$-semigroup with following multiplication given by

$$
\begin{array}{l|lll}
\cdot & a & b & c \\
\hline a & a & a & a \\
b & a & b & b \\
c & a & b & c
\end{array}
$$

We define a mapping $\phi: \mathcal{I}(S) \rightarrow \mathcal{I}(S) \cup\{\emptyset\}$ as follows: $\phi(A)=\emptyset$ for every $A \in \mathcal{I}(S)$. Clearly, $\{a\}$ and $\{a, b\}$ are $\phi$-quasi-prime ideal of an ordered $\mathcal{L} \mathcal{A}$-semigroup $S$.

Remark 1 It is easy to see that every quasi-prime ideal of an ordered $\mathcal{L} \mathcal{A}$-semigroup $S$ is a $\phi$-quasi-prime ideal of $S$.

The following example shows that the converse of Remark 1 is not true.

Example 2 Let $S=\{a, b, c\}$ be an ordered $\mathcal{L} \mathcal{A}$-semigroup with following multiplication given by

$$
\begin{array}{l|lll}
\cdot & a & b & c \\
\hline a & a & a & a \\
b & c & c & c \\
c & a & a & c
\end{array}
$$

Consider the proper left ideal $P=\{a, c\}$ of the ordered $\mathcal{L} \mathcal{A}$-semigroup $S$. Define $\phi: \mathcal{I}(S) \rightarrow$ $\mathcal{I}(S) \cup\{\varnothing\}$ by $\phi(A)=A$ for every $A \in \mathcal{I}(S)$. It is easy to see that $P$ is a $\phi$-quasi-prime ideal of $S$. Notice that $b \cdot b=c \in P$, but $b \notin P$. Therefore $P$ is not a quasi-prime ideal of $S$.

Let $A$ be a left ideal of an ordered $\mathcal{L} \mathcal{A}$-semigroup $S$ and let $\phi: \mathcal{I}(S) \rightarrow \mathcal{I}(S) \cup\{\emptyset\}$ be a function. Since $A-\phi(A)=A-(A \cap \phi(A))$ for $A \in \mathcal{I}(S)$, without loss of generality, we will assume that $\phi(A) \subseteq A$. Throughout this paper, as it is noted earlier, if $\phi$ is a function, then we always assume that $\phi(A) \subseteq A$.

Theorem 1 Let $A$ be a $\phi$-quasi-prime ideal of an ordered $\mathcal{L} \mathcal{A}$-semigroup $S$ with left identity. For each element s of $S-A$ if $\phi(A)$ is a quasi-prime ideal of $S$, then $(A: s]$ is a $\phi$-quasi-prime ideal of $S$ with $(\phi(A): s] \subseteq \phi(A: s]$. 
Proof Obviously, $(A: s]$ is a left ideal of $S$. Let $a$ and $b$ be any elements of $S$ such that $a b \in(A: s]-\phi(A: s]$. Since $(\phi(A): s] \subseteq \phi(A: s]$, we have $a(s b)=s(a b) \in A-\phi(A)$. By assumption, $a \in A$ or $b s \in A$. If $b s \in \phi(A)$, then $b \in \phi(A)$ or $s \in \phi(A)$. Now, if $b s \notin \phi(A)$, then $b s \in A-\phi(A)$. Then from hypothesis, $b \in A$ or $s \in A$. In any case, we have $a \in A$ or $b \in A$, which implies that $s a \in(s A] \subseteq(A]=A$ or $s b \in(s A] \subseteq(A]=A$. Consequently, $a \in(A: s]$ or $b \in(A: s]$ and hence $(A: s]$ is a $\phi$-quasi-prime ideal of $S$.

Remark 2 Let $A$ be a $\phi$-quasi-prime ideal of an ordered $\mathcal{L} \mathcal{A}$-semigroup $S$ and let $\phi(A)$ be a quasi-prime ideal of $S$ with $s_{1} \notin A, s_{2} \notin\left(A: s_{1}\right], s_{3} \notin\left(\left(A: s_{1}\right]: s_{2}\right], \ldots$ and $\left(\phi(A): s_{1}\right] \subseteq$ $\phi\left(A: s_{1}\right],\left(\left(\phi(A): s_{1}\right]: s_{2}\right] \subseteq \phi\left(\left(A: s_{1}\right]: s_{2}\right], \ldots$ Then $\left(A: s_{1}\right],\left(\left(A: s_{1}\right]: s_{2}\right], \ldots$ are $\phi$-quasi-prime ideals of $S$ and $A \subseteq\left(A: s_{1}\right] \subseteq\left(\left(A: s_{1}\right]: s_{2}\right] \subseteq \ldots$

In the following result, we give an equivalent definition of $\phi$-quasi-prime ideals in an ordered $\mathcal{L} \mathcal{A}$-semigroup.

Theorem 2 Let $S$ be an ordered $\mathcal{L} \mathcal{A}$-semigroup and let $\phi: \mathcal{I}(S) \rightarrow \mathcal{I}(S) \cup\{\emptyset\}$ be a function. The following conditions are equivalent:

1. A is a $\phi$-quasi-prime ideal of $S$.

2. For each an element $a$ of $S$ if $a \in S-A$, then $(A: a]=(\phi(A): a] \cup A$.

Proof First assume that $A$ is a $\phi$-quasi-prime ideal of $S$. It is easy to see that, $(\phi(A)$ : $a)] \cup A \subseteq(A: a]$. Let $b$ be an element of $S$ such that $b \in(A: a]$. Then we have, $a b \in A$. If $a b \notin \phi(A)$, then $a b \in A-\phi(A)$. Since $A$ is a $\phi$-quasi-prime ideal of $S$, we have $a \in A$ or $b \in A$. By assumption, $b \in A$ that is, $b \in(\phi(A): a] \cup A$. Now, if $a b \in \phi(A)$, then $b \in(\phi(A): a] \subseteq(\phi(A): a] \cup A$. In any case, we have $(A: a] \subseteq(\phi(A): a] \cup A$ and hence $(A: a]=(\phi(A): a] \cup A$.

Conversely, assume that 2 holds. Let $a$ and $b$ be any elements of $S$ such that $a b \in A-\phi(A)$. Then we have, $b \in(A: a]$ and $b \notin(\phi(A): a]$. If $a \in A$, then there is nothing to prove. Now, if $a \notin A$, then $(A: a]=(\phi(A): a] \cup A$. Since $b \in(A: a]$ and $b \notin(\phi(A): a]$, we have $b \in A$. Therefore $A$ is a $\phi$-quasi-prime ideal of $S$.

The following theorem characterize that quasi-prime ideals in terms of $\phi$-quasi-prime ideals of an ordered $\mathcal{L} \mathcal{A}$-semigroup $S$.

Theorem 3 Let $\phi: \mathcal{I}(S) \rightarrow \mathcal{I}(S) \cup\{\emptyset\}$ be a function and let $\phi(A)$ be a quasi-prime ideal of an ordered $\mathcal{L} \mathcal{A}$-semigroup $S$. Then $A$ is a $\phi$-quasi-prime ideal of $S$ if and only if $A$ is a quasi-prime ideal of $S$.

Proof First assume that $A$ is a quasi-prime ideal of $S$. Obviously, $A$ is a $\phi$-quasi-prime ideal of $S$.

Conversely, assume that $A$ is a $\phi$-quasi-prime ideal of $S$. Let $a$ and $b$ be any elements of $S$ such that $a b \in A$. If $a b \notin \phi(A)$, then $a b \in A-\phi(A)$. By assumption, $a \in A$ or $b \in A$. Now if $a b \in \phi(A)$, then $a \in A$ or $b \in A$. In any case, we have $A$ is a $\phi$-quasi-prime ideal of $S$.

Now we introduce the notion of a $\phi$-zero in an ordered $\mathcal{L} \mathcal{A}$-semigroup.

Definition 2 Let $\phi: \mathcal{I}(S) \rightarrow \mathcal{I}(S) \cup\{\varnothing\}$ be a function and let $A$ be a $\phi$-quasi-prime ideal of an ordered $\mathcal{L} \mathcal{A}$-semigroup $S$. An order pair $(a, b)$, where $a, b \in S$ is a $\phi$-zero if

1. $a b \in \phi(A)$, 
2. $a \notin A$ and $b \notin A$.

Remark 3 Note that a proper left ideal $A$ of an ordered $\mathcal{L} \mathcal{A}$-semigroup $S$ is a $\phi$-quasi-prime ideal of $S$ that is not a quasi-prime ideal of $S$ if and only if $A$ has a $\phi$-zero $(a, b)$ for some $a, b \in S$.

Theorem 4 Let $\phi: \mathcal{I}(S) \rightarrow \mathcal{I}(S) \cup\{\emptyset\}$ be a function and let $A$ be a $\phi$-quasi-prime ideal of an ordered $\mathcal{L} \mathcal{A}$-semigroup $S$. Suppose that $B$ is a left ideal of $S$ and $a \in S$ such that $a B \subseteq A$. If for every an element $b$ of $S$ such that $(a, b)$ is not a $\phi$-zero of $A$, then $a \in A$ or $B \subseteq A$.

Proof Assume, $a \notin A$ and $B \nsubseteq A$. Then there exists an element $c \in B$ such that $c \notin A$. If $a c \notin \phi(A)$, then $a c \in A-\phi(A)$. Since $A$ is a $\phi$-quasi-prime ideal of $S$, we have $a \in A$ or $c \in A$. Next, let $a c \in \phi(A)$. By hypothesis, $a \in A$ or $c \in A$. In any case, we have $a \in A$ or $c \in A$, which is a contradiction. Hence, $a \in A$ or $B \subseteq A$.

Theorem 5 Let $\phi: \mathcal{I}(S) \rightarrow \mathcal{I}(S) \cup\{\emptyset\}$ be a function and let $A$ be a $\phi$-quasi-prime ideal of an ordered $\mathcal{L} \mathcal{A}$-semigroup $S$. For each elements $a, b \in S$ if $(a, b)$ is a $\phi$-zero of $A$, then $a A \subseteq \phi(A)$.

Proof Let $a$ be an element of $S$ such that $a A \nsubseteq \phi(A)$. Then there exists an element $c$ of $A$ such that $a c \notin \phi(A)$. Thus we have, $a(b \cup c)=(a b) \cup(a c) \nsubseteq \phi(A)$, which implies that $a(b \cup c) \subseteq A-\phi(A)$. Since $A$ is a $\phi$-quasi-prime ideal of $S$, we have $a \in A$ or $b \cup c \subseteq A$. Therefore, $a \in A$ or $b \in A$, which is a contradiction. Consequently, $a A \subseteq \phi(A)$.

Theorem 6 Let $\phi: \mathcal{I}(S) \rightarrow \mathcal{I}(S) \cup\{\emptyset\}$ be a function. If $A$ is a $\phi$-quasi-prime ideal of an ordered $\mathcal{L} \mathcal{A}$-semigroup $S$ that is not a quasi-prime ideal, then $A^{2}=\phi(A)$.

Proof Since $A$ is a $\phi$-quasi-prime ideal of $S$ that is not a quasi-prime ideal, we have $A$ has a $\phi$-zero $(a, b)$ for some $a, b \in S$ by Remark 3. Assume, $c d \notin \phi(A)$ for some $c, d \in A$. Then we have, $(a \cup c)(b \cup d)=a b \cup c b \cup a d \cup c d \nsubseteq \phi(A)$ by Theorem 5. This implies that, $(a \cup c)(b \cup d) \subseteq A-\phi(A)$. By assumption, $a \cup c \subseteq A$ or $b \cup d \subseteq A$. Therefore, $a \in A$ or $b \in A$, which is a contradiction. Hence, $A^{2}=\phi(A)$.

\section{$3 \phi_{\alpha}$-quasi-prime ideals}

In this section, we introduce the concept of $\phi$-quasi-prime, $\phi_{\emptyset}$-quasi-prime, $\phi_{n \geq 1}$-quasi-prime and $\phi_{\omega}$-quasi-prime ideals of ordered $\mathcal{L} \mathcal{A}$-semigroups and study some basic properties of $\phi$-quasi-prime, $\phi_{\emptyset}$-quasi-prime, $\phi_{n \geq 1}$-quasi-prime and $\phi_{\omega}$-quasi-prime ideals of ordered $\mathcal{L} \mathcal{A}$ semigroups. Our starting points are the following definitions:

Definition 3 Let $\alpha \in \mathbf{Z}^{+} \cup\{\omega\} \cup\{\emptyset\}$ and let $\phi_{\alpha}: \mathcal{I}(S) \rightarrow \mathcal{I}(S) \cup\{\emptyset\}$ be a function where $\mathcal{I}(S)$ is a set of all left ideals of an ordered $\mathcal{L} \mathcal{A}$-semigroup $S$. A proper left ideal $A$ of $S$ is called a $\phi_{\alpha}$-quasi-prime ideal if for each $a, b \in S$ with $a b \in A-\phi_{\alpha}(A)$, then $a \in A$ or $b \in A$.

Let $A$ be a $\phi_{\alpha}$-quasi-prime ideal of an ordered $\mathcal{L} \mathcal{A}$-semigroup $S$.

- If $\phi_{\alpha}(A)=\emptyset$ for every $A \in \mathcal{I}(S)$, then we say that $\phi_{\alpha}=\phi_{\emptyset}$ and $A$ is called a $\phi_{\emptyset}$-quasiprime ideal of $S$ and hence $A$ is a quasi-prime ideal of $S$.

- If $\phi_{\alpha}(A)=A$ for every $A \in \mathcal{I}(S)$, then we say that $\phi_{\alpha}=\phi_{1}$ and $A$ is called a $\phi_{1}$-quasiprime ideal of $S$. 
- If $\phi_{\alpha}(A)=A^{2}$ for every $A \in \mathcal{I}(S)$, then we say that $\phi_{\alpha}=\phi_{2}$ and $A$ is called a $\phi_{n}$-quasi-prime ideal of $S$, and hence $A$ is an almost quasi-prime ideal of $S$.

- If $\phi_{\alpha}(A)=A^{m}$ for every $A \in \mathcal{I}(S)$, then we say that $\phi_{\alpha}=\phi_{m \geq 3}$ and $A$ is called a $\phi_{m}$-quasi-prime ideal of $S$, and hence $A$ is a $m$-quasi-prime ideal of $S$.

- If $\phi_{\alpha}(A)=\bigcap_{i=1}^{\infty} A^{i}$ for every $A \in \mathcal{I}(S)$, then we say that $\phi_{\alpha}=\phi_{\omega}$ and $A$ is called a $\phi_{\omega}$-quasi-prime ideal of $S$, and hence $A$ is an $\omega$-quasi-prime ideal of $S$.

Remark 4 Let $\alpha \in \mathbf{Z}^{+} \cup\{\omega\} \cup\{\emptyset\}$ and let $\phi_{\alpha}: \mathcal{I}(S) \rightarrow \mathcal{I}(S) \cup\{\emptyset\}$ be a function.

1. A left ideal $A$ of an ordered $\mathcal{L} \mathcal{A}$-semigroup $S$ is a $\phi_{\emptyset}$-quasi-prime ideal of $S$ if and only if $A$ is a quasi-prime ideal of $S$.

2. A left ideal $A$ of an ordered $\mathcal{L} \mathcal{A}$-semigroup $S$ is a $\phi_{1}$-quasi-prime ideal of $S$ if and only if $A$ is a proper left ideal of $S$.

3. If $A$ is a quasi-prime ideal of an ordered $\mathcal{L} \mathcal{A}$-semigroup $S$, then $A$ is a $\phi_{\alpha}$-quasi-prime ideal of $S$.

We start with our main result in which we give a characterization of $\phi_{\alpha}$-quasi-prime ideals in ordered $\mathcal{L} \mathcal{A}$-semigroups. For that, we need the following proposition.

Proposition 1 Let $S$ be an ordered $\mathcal{L} \mathcal{A}$-semigroup and let $\phi, \varphi: \mathcal{I}(S) \rightarrow \mathcal{I}(S) \cup\{\emptyset\}$ be two functions. Then the following properties hold:

1. If $A$ is a $\phi$-quasi-prime ideal of $S$ such that $\phi \leq \varphi$, then $A$ is a $\varphi$-quasi-prime ideal of $S$.

2. If $A$ is a quasi-prime ideal of $S$, then $A$ is a $\phi_{\omega}$-quasi-prime ideal of $S$.

3. If $A$ is a $\omega$-quasi-prime ideal of $S$, then $A$ is a m-quasi-prime ideal of $S$.

4. If $A$ is an almost quasi-prime ideal of $S$, then $A$ is a $\phi_{1}$-quasi-prime ideal of $S$.

Proof 1 . Let $a$ and $b$ be any elements of $S$ such that $a b \in A-\varphi(A)$. Since $\phi \leq \varphi$, we have $\phi(A) \subseteq \varphi(A)$. Then we have, $a b \in A-\varphi(A) \subseteq A-\phi(A)$. Since $A$ is a $\phi$-quasi-prime ideal of $S$, we have $a \in A$ or $b \in A$. Hence $A$ is a $\varphi$-quasi-prime ideal of $S$.

2 - 4. It are obvious.

Remark 5 Let $S$ be an ordered $\mathcal{L} \mathcal{A}$-semigroup and let $\mathcal{I}(S)$ be a set of all left ideals of $S$. It is easy to see that, $\phi_{\emptyset} \leq \phi_{\omega} \leq \ldots \leq \phi_{n+1} \leq \phi_{n} \leq \ldots \leq \phi_{2} \leq \phi_{1}$.

Let $S_{1}$ and $S_{2}$ be two ordered $\mathcal{L} \mathcal{A}$-semigroups. Then $S_{1} \times S_{2}$ is an ordered $\mathcal{L} \mathcal{A}$-semigroup and for each left ideal of $S_{1} \times S_{2}$ is of the form $A_{1} \times A_{2}$ for some left ideals $A_{1}$ and $A_{2}$ of $S_{1}$ and $S_{2}$, respectively.

Next we show that, $S_{1} \times \ldots \times S_{i-1} \times A_{i} \times S_{i+1} \times \ldots \times S_{k}$ is a $\phi$-quasi-prime ideal of $S_{1} \times \ldots \times S_{k}$ if and only if $A_{i}$ is a $\psi_{i}$-quasi-prime ideal of $S_{i}$. First, we would like to show that, $A_{1} \times S_{2}$ is a $\phi$-quasi-prime ideal of $S_{1} \times S_{2}$ if and only if $A_{1}$ is a $\psi_{1}$-quasi-prime ideal of $S_{1}$.

Theorem 7 Let $S_{1}$ and $S_{2}$ be two ordered $\mathcal{L} \mathcal{A}$-semigroups with left identities and let $\psi_{i}$ : $\mathcal{I}\left(S_{i}\right) \rightarrow \mathcal{I}\left(S_{i}\right) \cup\{\emptyset\}$ be a function with $\phi=\psi_{1} \times \psi_{2}$. Then the following conditions are equivalent:

1. $A_{1} \times S_{2}$ is a $\phi$-quasi-prime ideal of $S_{1} \times S_{2}$.

2. (a) $A_{1}$ is a $\psi_{1}$-quasi-prime ideal of $S_{1}$ where $\psi_{2}\left(S_{2}\right) \neq S_{2}$.

(b) For each elements $\left(a_{1}, b_{1}\right),\left(a_{2}, b_{2}\right)$ of $S_{1} \times S_{2}$ such that $a_{1} a_{2} \in \psi_{1}\left(A_{1}\right)$ if $b_{1} \in$ $S_{2}-\left(\psi_{2}\left(S_{2}\right): S_{2}\right]$, then $a_{1} \in A_{1}$ or $a_{2} \in A_{1}$. 
Proof First assume that $A_{1} \times S_{2}$ is a $\phi$-quasi-prime ideal of $S_{1} \times S_{2}$.

(a) Let $a_{1}$ and $a_{2}$ be any elements of $S_{1}$ such that $a_{1} a_{2} \in A_{1}-\psi_{1}\left(A_{1}\right)$. Then we have, $\left(a_{1}, e\right)\left(a_{2}, e\right)=\left(a_{1} a_{2}, e\right) \in A_{1} \times S_{2}-\psi_{1}\left(A_{1}\right) \times \psi_{2}\left(S_{2}\right)=A_{1} \times S_{2}-\phi\left(A_{1} \times S_{2}\right)$. By assumption, $\left(a_{1}, e\right) \in A_{1} \times S_{2}$ or $\left(a_{2}, e\right) \in A_{1} \times S_{2}$. Therefore, $a_{1} \in A_{1}$ or $a_{2} \in A_{1}$ and hence $A_{1}$ is a $\psi_{1}$-quasi-prime ideal of $S_{1}$.

(b) Let $\left(a_{1}, b_{1}\right)$, and $\left(a_{2}, b_{2}\right)$ be any elements of $S_{1} \times S_{2}$ be such that $a_{1} a_{2} \in \psi_{1}\left(A_{1}\right)$ and $a_{1}, a_{2} \notin A_{1}$. In fact, since $b_{1} \in S_{2}-\left(\psi_{2}\left(S_{2}\right): S_{2}\right]$, there exists an element $b_{2}$ of $S_{2}$ such that $b_{2} b_{1} \notin \psi_{2}\left(S_{2}\right)$. Thus, $\left(a_{1}, b_{2}\right)\left(a_{2}, b_{1}\right)=\left(a_{1} a_{2}, b_{2} b_{1}\right) \in A_{1} \times S_{2}-$ $\psi_{1}\left(A_{1}\right) \times \psi_{2}\left(S_{2}\right)=A_{1} \times S_{2}-\phi\left(A_{1} \times S_{2}\right)$. Then by part (a), i.e., $\left(a_{1}, b_{2}\right) \in A_{1} \times S_{2}$ or $\left(a_{2}, b_{1}\right) \in A_{1} \times S_{2}$. Therefore, $a_{1} \in A_{1}$ or $a_{2} \in A_{1}$, which is a contradiction. Consequently, $b_{1} \in\left(\psi_{2}\left(S_{2}\right): S_{2}\right]$.

Assume that 2 holds. Let $\left(a_{1}, b_{1}\right)$ and $\left(a_{2}, b_{2}\right)$ be any elements of $S_{1} \times S_{2}$ be such that $\left(a_{1} a_{2}, b_{1} b_{2}\right)=\left(a_{1}, b_{1}\right)\left(a_{2}, b_{2}\right) \in A_{1} \times S_{2}-\phi\left(A_{1} \times S_{2}\right)=A_{1} \times S_{2}-\psi_{1}\left(A_{1}\right) \times \psi_{2}\left(S_{2}\right)$. If $a_{1} a_{2} \notin \psi_{1}\left(A_{1}\right)$, then $a_{1} a_{2} \in A_{1}-\psi_{1}\left(S_{1}\right)$. Then by part (a), $a_{1} \in A_{1}$ or $a_{2} \in A_{1}$. Thus, $\left(a_{1}, b_{1}\right) \in A_{1} \times S_{2}$ or $\left(a_{2}, b_{2}\right) \in A_{1} \times S_{2}$ and thus we are done. If $a_{1} a_{2} \in \psi_{1}\left(A_{1}\right)$, then $b_{1} b_{2} \notin \psi_{2}\left(S_{2}\right)$, which implies that $b_{2} \notin\left(\psi_{2}\left(S_{2}\right): S_{2}\right]$. Hence by part (b), $a_{1} \in A_{1}$ or $a_{2} \in A_{1}$. Therefore, $\left(a_{1}, b_{1}\right) \in A_{1} \times S_{2}$ or $\left(a_{2}, b_{2}\right) \in A_{1} \times S_{2}$ and hence $A_{1} \times S_{2}$ is a $\phi$-quasi-prime ideal of $S_{1} \times S_{2}$.

The following theorem can be seen in a similar way as in the proof of Theorem 7 .

Theorem 8 Let $S_{1}$ and $S_{2}$ be two ordered $\mathcal{L} \mathcal{A}$-semigroups with left identities and let $\psi_{i}$ : $\mathcal{I}\left(S_{i}\right) \rightarrow \mathcal{I}\left(S_{i}\right) \cup\{\emptyset\}$ be a function with $\phi=\psi_{1} \times \psi_{2}$. Then the following conditions are equivalent:

1. $S_{1} \times A_{2}$ is a $\phi$-quasi-prime ideal of $S_{1} \times S_{2}$.

2. (a) $A_{2}$ is a $\psi_{2}$-quasi-prime ideal of $S_{2}$ where $\psi_{1}\left(S_{1}\right) \neq S_{1}$.

(b) For each elements $\left(a_{1}, b_{1}\right),\left(a_{2}, b_{2}\right)$ of $S_{1} \times S_{2}$ such that $b_{1} b_{2} \in \psi_{2}\left(A_{2}\right)$ if $a_{1} \in$ $S_{1}-\left(\psi_{1}\left(S_{1}\right): S_{1}\right]$, then $b_{1} \in A_{2}$ or $b_{2} \in A_{2}$.

The proof of the next result is similar to that of Theorem 7 .

Theorem 9 Let $S_{i}$ be a ordered $\mathcal{L} \mathcal{A}$-semigroup with left identity and let $\psi_{i}: \mathcal{I}\left(S_{i}\right) \rightarrow$ $\mathcal{I}\left(S_{i}\right) \cup\{\emptyset\}$ be a function with $\phi=\psi_{1} \times \ldots \times \psi_{k}$. Then the following conditions are equivalent:

1. $S_{1} \times \ldots \times S_{i-1} \times A_{i} \times S_{i+1} \times \ldots \times S_{k}$ is a $\phi$-quasi-prime ideal of $S_{1} \times \ldots \times S_{k}$.

2. (a) $A_{i}$ is a $\psi_{i}$-quasi-prime deal of $S_{i}$ where $\psi_{j}\left(S_{j}\right) \neq S_{j}$.

(b) For each elements $\left(a_{(1,1)}, \ldots, a_{(k, 1)}\right),\left(a_{(1,2)}, \ldots, a_{(k, 2)}\right)$ of $S_{1} \times \ldots \times S_{k}$ such that $a_{(1, i)} a_{(2, i)} \in \psi_{i}\left(A_{i}\right)$ if $a_{(j, 1)} \in S_{j}-\left(\psi_{j}\left(S_{j}\right): S_{j}\right)$ for all $j \in\{1, \ldots, k\}-\{i\}$, then $a_{(1, i)} \in A_{i}$ or $a_{(2, i)} \in A_{i}$.

Recall that an element 0 of an ordered $\mathcal{L} \mathcal{A}$-semigroup $S$ is called a left zero element of $S$ if $0 s \leq 0$ for any $s \in S$.

Let $S$ be an ordered $\mathcal{L} \mathcal{A}$-semigroup with left zero. If $\phi_{\alpha}(A)=\{0\}$ for every $A \in \mathcal{I}(S)$, then we say that $\phi_{\alpha}=\phi_{0}$ and $A$ is called a $\phi_{0}$-quasi-prime ideal of $S$, and hence $A$ is a weakly quasi-prime ideal of $A$.

As a simple consequence of Theorem 6, we give the following result.

Theorem 10 Let $\phi: \mathcal{I}(S) \rightarrow \mathcal{I}(S) \cup\{\emptyset\}$ be a function and let A be a left ideal of an ordered $\mathcal{L} \mathcal{A}$-semigroup $S$ with left zero that is not a quasi-prime ideal. If $A$ is a weakly quasi-prime ideal of $S$, then $A^{2}=\{0\}$. 
Next we show that, if $A_{i}$ is a $\left(\psi_{i}\right)_{0}$-quasi-prime ideal of $S_{i}$, then $S_{1} \times S_{2} \times \ldots \times S_{i-1} \times$ $A_{i} \times S_{i+1} \times \ldots \times S_{k}$ is a $\phi$-quasi-prime ideal of an ordered $\mathcal{L} \mathcal{A}$-semigroup $S_{1} \times S_{2} \times \ldots \times S_{k}$ if $S_{1} \times \ldots \times S_{i-1} \times\{0\} \times S_{i+1} \times \ldots \times S_{k} \subseteq \phi\left(S_{1} \times \ldots \times S_{i-1} \times A_{i} \times S_{i+1} \times \ldots \times S_{k}\right)$. First, we would like to show that, $A_{1}$ is a $\left(\psi_{1}\right)_{0}$-quasi-prime ideal of an ordered $\mathcal{L} \mathcal{A}$-semigroup $S_{1}$, then $A_{1} \times S_{2}$ is a $\phi$-quasi-prime ideal if $\{0\} \times S_{2} \subseteq \phi\left(A_{1} \times S_{2}\right)$.

Theorem 11 Let $S_{1}$ and $S_{2}$ be two ordered $\mathcal{L} \mathcal{A}$-semigroups with left zeroes and let $\psi_{i}$ : $\mathcal{I}\left(S_{i}\right) \rightarrow \mathcal{I}\left(S_{i}\right) \cup\{\emptyset\}$ be a function with $\phi=\psi_{1} \times \psi_{2}$. If $A_{1}$ is a weakly quasi-prime ideal of $S_{1}$ such that $\{0\} \times S_{2} \subseteq \phi\left(A_{1} \times S_{2}\right)$, then $A_{1} \times S_{2}$ is a $\phi$-quasi-prime ideal of $S_{1} \times S_{2}$.

Proof Let $\left(a_{1}, b_{1}\right)$ and $\left(a_{2}, b_{2}\right)$ be any elements of $S_{1} \times S_{2}$ be such that

$$
\left(a_{1}, b_{1}\right)\left(a_{2}, b_{2}\right) \in A_{1} \times S_{2}-\phi\left(A_{1} \times S_{2}\right) .
$$

In fact, since $\{0\} \times S_{2} \subseteq \phi\left(A_{1} \times S_{2}\right)$, we have $\left(a_{1} a_{2}, b_{1} b_{2}\right)=\left(a_{1}, b_{1}\right)\left(a_{2}, b_{2}\right) \notin\{0\} \times S_{2}$, which means that $a_{1} a_{2} \neq 0$. Then we have, $a_{1} a_{2} \in A_{1}-\left(\psi_{1}\right)_{0}\left(A_{1}\right)$. Since $A_{1}$ is a weakly quasi-prime ideal of $S_{1}$, we have $a_{1} \in A_{1}$ or $a_{2} \in A_{1}$. Therefore, $\left(a_{1}, b_{1}\right) \in A_{1} \times S_{2}$ or $\left(a_{2}, b_{2}\right) \in A_{1} \times S_{2}$ and hence $A_{1} \times S_{2}$ is a $\phi$-quasi-prime ideal of $S_{1} \times S_{2}$.

From Theorem 11 we can easily obtain the following theorem.

Theorem 12 Let $S_{1}$ and $S_{2}$ be two ordered $\mathcal{L} \mathcal{A}$-semigroups with left zeroes and let $\psi_{i}$ : $\mathcal{I}\left(S_{i}\right) \rightarrow \mathcal{I}\left(S_{i}\right) \cup\{\emptyset\}$ be a function with $\phi=\psi_{1} \times \psi_{2}$. If $A_{2}$ is a weakly quasi-prime ideal of $S_{2}$ such that $S_{1} \times\{0\} \subseteq \phi\left(S_{1} \times A_{2}\right)$, then $S_{1} \times A_{2}$ is a $\phi$-quasi-prime ideal of $S_{1} \times S_{2}$.

From Theorems 11, 12 we can easily obtain the following theorem.

Theorem 13 Let $S_{i}$ be an ordered $\mathcal{L} \mathcal{A}$-semigroup with left zero and let $\psi_{i}: \mathcal{I}\left(S_{i}\right) \rightarrow \mathcal{I}\left(S_{i}\right) \cup$ $\{\emptyset\}$ be a function with $\phi=\psi_{1} \times \ldots \times \psi_{k}$ and $S_{1} \times \ldots \times S_{i-1} \times\{0\} \times S_{i+1} \times \ldots \times S_{k} \subseteq$ $\phi\left(S_{1} \times \ldots \times S_{i-1} \times A_{i} \times S_{i+1} \times \ldots \times S_{k}\right)$. Then $A_{i}$ is a weakly quasi-prime ideal of $S_{i}$ if and only if $S_{1} \times S_{2} \times \ldots \times S_{i-1} \times A_{i} \times S_{i+1} \times \ldots \times S_{k}$ is a $\phi$-quasi-prime ideal of $S_{1} \times S_{2} \times \ldots \times S_{k}$.

Next, let $S$ be an ordered $\mathcal{L} \mathcal{A}$-semigroup. Clearly, every quasi-prime ideal of $S$ is $\phi$ quasi-prime ideal, but the converse does not necessarily hold. In Theorem 14 and Corollary 1 provide some conditions under which a $\phi$-quasi-prime ideal is a quasi-prime ideal in an ordered $\mathcal{L} \mathcal{A}$-semigroup.

Theorem 14 Let $\phi, \phi_{3}: \mathcal{I}(S) \rightarrow \mathcal{I}(S) \cup\{\emptyset\}$ be two functions and let $A$ be a $\phi$-quasi-prime ideal of an ordered $\mathcal{L} \mathcal{A}$-semigroup $S$. If $\phi_{2} \not \leq \phi$, then $A$ is a quasi-prime ideal of $S$.

Proof Let $a_{1}$ and $a_{2}$ be any elements of $S$ such that $a_{1} a_{2} \in A$. If $a_{1} a_{2} \notin \phi(A)$, then $a_{1} a_{2} \in A-\phi(A)$. Since $A$ is a $\phi$-quasi-prime ideal of $S$, we have $a_{1} \in A$ or $a_{2} \in A$. Next, let $a_{1} a_{2}$ be an element of $\phi(A)$. Since $\phi_{2} \not \leq \phi$, we have $A^{2} \nsubseteq \phi(A)$. Then there exist elements $b_{1}$ and $b_{2}$ of $A$ such that $b_{1} b_{2} \notin \phi(A)$, which means that $\left(a_{1} \cup b_{1}\right)\left(a_{2} \cup b_{2}\right)=$ $a_{1} b_{1} \cup a_{2} b_{1} \cup a_{1} b_{2} \cup a_{2} b_{2} \subseteq A-\phi(A)$. By hypothesis, $a_{1} \cup b_{1} \subseteq A$ or $a_{2} \cup b_{2} \subseteq A$. Therefore, $a_{1} \in A$ or $a_{2} \in A$ and hence $A$ is a quasi-prime ideal of $S$.

In the following theorem, we give a sort of consequences whose proof is similar to those of quasi-prime ideals in ordered $\mathcal{L} \mathcal{A}$-semigroups.

Corollary 1 Let $\phi_{n}: \mathcal{I}(S) \rightarrow \mathcal{I}(S) \cup\{\emptyset\}$ be a function and let $A$ be a weakly quasi-prime ideal of an ordered $\mathcal{L} \mathcal{A}$-semigroup $S$ with left zero. If $\phi_{2} \neq \phi_{0}$, then $A$ is a quasi-prime ideal of $S$. 
Proof Similar to the proof of Theorem 14.

Let $S_{i}$ be an ordered $\mathcal{L} \mathcal{A}$-semigroup. For each elements $k, n$ of $\mathbf{Z}^{+}$such that $k \geq 2, n \geq$ $1,\left(\psi_{i}\right)_{n}: \mathcal{I}\left(S_{i}\right) \rightarrow \mathcal{I}\left(S_{i}\right) \cup\{\emptyset\}$ and let $\phi_{(k, n)}=\left(\psi_{1}\right)_{n} \times\left(\psi_{2}\right)_{n} \times \ldots \times\left(\psi_{k}\right)_{n}$.

Theorem 15 If $A_{1}$ is a weakly quasi-prime ideal of an ordered $\mathcal{L} \mathcal{A}$-semigroup $S_{1}$ with left zero such that $\left(\psi_{2}\right)_{2}\left(S_{2}\right)=S_{2}$, then $A_{1} \times S_{2}$ is a $\phi_{(2,2)}$-quasi-prime ideal of $S_{1} \times S_{2}$.

Proof If $A_{1}$ is a quasi-prime ideal of $S_{1}$, then $A_{1} \times S_{2}$ is a quasi-prime ideal of $S_{1} \times S_{2}$. Obviously, $A_{1} \times S_{2}$ is a $\phi_{(2,2)}$-quasi-prime ideal of $S_{1} \times S_{2}$. Assume that $A_{1}$ is not a quasiprime ideal of $S_{1}$. Then by Corollary $1,\left(\psi_{1}\right)_{2} \leq\left(\psi_{1}\right)_{0}$, which implies that $\left(A_{1}\right)^{2}=\{0\}$. By assumption,

$$
\begin{aligned}
\phi_{(2,2)}\left(A_{1} \times S_{2}\right) & =\left(\psi_{1}\right)_{2} \times\left(\psi_{2}\right)_{2}\left(A_{1} \times S_{2}\right) \\
& =\left(\psi_{1}\right)_{2}\left(A_{1}\right) \times\left(\psi_{2}\right)_{2}\left(S_{2}\right) \\
& =\{0\} \times S_{2} .
\end{aligned}
$$

It follows from Theorem 11 that $A_{1} \times S_{2}$ is a $\phi_{(2,2)}$-quasi-prime ideal of $S_{1} \times S_{2}$.

From Theorem 15 we can easily obtain the following theorem.

Theorem 16 If $A_{2}$ is a weakly quasi-prime ideal of an ordered $\mathcal{L} \mathcal{A}$-semigroup $S_{2}$ with left zero such that $\left(\psi_{1}\right)_{2}\left(S_{1}\right)=S_{1}$, then $S_{1} \times A_{2}$ is a $\phi_{(2,2)}$-quasi-prime ideal of $S_{1} \times S_{2}$.

From Theorems 15, 16 we can easily obtain the following theorem.

Theorem 17 If $A_{i}$ is a weakly quasi-prime ideal of an ordered $\mathcal{L} \mathcal{A}$-semigroup $S_{i}$ with left zero such that $\left(\psi_{j}\right)_{2}\left(S_{j}\right)=S_{j}$, then $S_{1} \times S_{2} \times \ldots \times S_{i-1} \times A_{i} \times S_{i+1} \times \ldots \times S_{k}$ is a $\phi_{(k, 2)}$-quasi-prime ideal of $S_{1} \times \ldots \times S_{k}$.

Next we show that, if $A_{i}$ is a quasi-prime ideal of an ordered $\mathcal{L} \mathcal{A}$-semigroup $S_{i}$, then $S_{1} \times S_{2} \times \ldots \times S_{i-1} \times A_{i} \times S_{i+1} \times \ldots \times S_{k}$ is a $\phi$-quasi-prime ideal of $S_{1} \times \ldots \times S_{2}$ if $\psi_{j}\left(S_{j}\right) \neq S_{j}$. First, we would like to show that, $A_{1}$ is a quasi-prime ideal of an ordered $\mathcal{L} \mathcal{A}$-semigroup $S_{1}$, then $A_{1} \times S_{2}$ is a $\phi$-quasi-prime ideal of $S_{1} \times S_{2}$ if $\psi_{2}\left(S_{2}\right) \neq S_{2}$.

Theorem 18 Let $S_{1}$ and $S_{2}$ be two ordered $\mathcal{L} \mathcal{A}$-semigroups with left identities and let $\psi_{i}$ : $\mathcal{I}\left(S_{i}\right) \rightarrow \mathcal{I}\left(S_{i}\right) \cup\{\emptyset\}$ be a function such that $\phi=\psi_{1} \times \psi_{2}$. Then the following conditions are equivalent:

1. $A_{1}$ is a quasi-prime ideal of $S_{1}$.

2. $A_{1} \times S_{2}$ is a quasi-prime ideal of $S_{1} \times S_{2}$.

3. $A_{1} \times S_{2}$ is a $\phi$-quasi-prime ideal of $S_{1} \times S_{2}$ where $\psi_{2}\left(S_{2}\right) \neq S_{2}$.

Proof $(1 \Rightarrow 2)$. Assume that $A_{1}$ is a quasi-prime ideal of $S_{1}$. Let $\left(a_{1}, b_{1}\right)$ and $\left(a_{2}, b_{2}\right)$ be any elements of $S_{1} \times S_{2}$ be such that $\left(a_{1} a_{2}, b_{1} b_{2}\right)=\left(a_{1}, b_{1}\right)\left(a_{2}, b_{2}\right) \in A_{1} \times S_{2}$, which implies that $a_{1} a_{2} \in A_{1}$. By assumption, $a_{1} \in A_{1}$ or $a_{2} \in A_{1}$. Therefore, $\left(a_{1}, b_{1}\right) \in A_{1} \times S_{2}$ or $\left(a_{2}, b_{2}\right) \in A_{1} \times S_{2}$. Consequently, $A_{1} \times S_{2}$ is a quasi-prime ideal of $S_{1} \times S_{2}$.

$(2 \Rightarrow 3)$. It is obvious.

$\left(3 \Rightarrow 1\right.$ ). Assume that 3 holds. Let $a_{1}$ and $a_{2}$ be any elements of $S_{1}$ be such that $a_{1} a_{2} \in A_{1}$. Since $\psi_{2}\left(S_{2}\right) \neq S_{2}$, there exists an element $c$ of $S_{2}$ such that $c \notin \psi_{2}\left(S_{2}\right)$. In fact, since $\left(a_{1}, e\right)\left(a_{2}, c\right)=\left(a_{1} a_{2}, c\right) \notin A_{1} \times \psi_{2}\left(S_{2}\right)$ and $\phi\left(A_{1} \times S_{2}\right)=\left(\psi_{1} \times \psi_{2}\right)\left(A_{1} \times S_{2}\right) \subseteq$ $A_{1} \times \psi_{2}\left(S_{2}\right)$, we have $\left(a_{1}, e\right)\left(a_{2}, c\right) \notin \phi\left(A_{1} \times S_{2}\right)$, which means that $\left(a_{1}, e\right)\left(a_{2}, c\right) \in A_{1} \times$ $S_{2}-\phi\left(A_{1} \times S_{2}\right)$. Since $A_{1} \times S_{2}$ is a $\phi$-quasi-prime ideal of $S_{1} \times S_{2}$, we have $\left(a_{1}, e\right) \in A_{1} \times S_{2}$ or $\left(a_{2}, c\right) \in A_{1} \times S_{2}$. Therefore, $a_{1} \in A_{1}$ or $a_{2} \in A_{1}$ and hence $A_{1}$ is a quasi-prime ideal of $S_{1}$. 
From Theorem 18 we can easily obtain the following theorem.

Theorem 19 Let $S_{1}$ and $S_{2}$ be two ordered $\mathcal{L} \mathcal{A}$-semigroups with left identities and let $\psi_{i}$ : $\mathcal{I}\left(S_{i}\right) \rightarrow \mathcal{I}\left(S_{i}\right) \cup\{\emptyset\}$ be a function such that $\phi=\psi_{1} \times \psi_{2}$. Then the following conditions are

1. $A_{2}$ is a quasi-prime ideal of $S_{2}$.

2. $S_{1} \times A_{2}$ is a quasi-prime ideal of $S_{1} \times S_{2}$.

3. $S_{1} \times A_{2}$ is a $\phi$-quasi-prime ideal of $S_{1} \times S_{2}$, where $\psi_{1}\left(S_{1}\right) \neq S_{1}$.

From Theorems 18, 19 we can easily obtain the following theorem.

Theorem 20 Let $S_{i}$ be a ordered $\mathcal{L} \mathcal{A}$-semigroup with left identity and let $\psi_{i}: \mathcal{I}\left(S_{i}\right) \rightarrow$ $\mathcal{I}\left(S_{i}\right) \cup\{\emptyset\}$ be a function such that $\phi=\psi_{1} \times \ldots \times \psi_{k}$. Then the following conditions are equivalent:

1. $A_{i}$ is a quasi-prime ideal of $S_{i}$.

2. $S_{1} \times S_{2} \times \ldots \times S_{i-1} \times A_{i} \times S_{i+1} \times \ldots \times S_{k}$ is a quasi-prime ideal of $S_{1} \times \ldots \times S_{k}$.

3. $S_{1} \times S_{2} \times \ldots \times S_{i-1} \times A_{i} \times S_{i+1} \times \ldots \times S_{k}$ is a $\phi$-quasi-prime ideal of $S_{1} \times \ldots \times S_{2}$ with $\psi_{j}\left(S_{j}\right) \neq S_{j}$.

Next, we show that if $A_{i}$ is a $\psi_{i}$-quasi-prime ideal of an ordered $\mathcal{L} \mathcal{A}$-semigroup $S_{i}$, then $S_{1} \times S_{2} \times \ldots \times S_{i-1} \times A_{i} \times S_{i+1} \times \ldots \times S_{k}$ is a $\phi$-quasi-prime ideal of $S_{1} \times \ldots \times S_{k}$ if $\psi_{j}\left(S_{j}\right)=S_{j}$. First, we would like to show that, $A_{1}$ is a $\psi_{1}$-quasi-prime ideal of an ordered $\mathcal{L} \mathcal{A}$-semigroup $S_{1}$, then $A_{1} \times S_{2}$ is a $\phi$-quasi-prime ideal of $S_{1} \times S_{2}$, if $\psi_{2}\left(S_{2}\right)=S_{2}$.

Theorem 21 Let $S_{1}$ and $S_{2}$ be two ordered $\mathcal{L} \mathcal{A}$-semigroups with left identities and let $\psi_{i}$ : $\mathcal{I}\left(S_{i}\right) \rightarrow \mathcal{I}\left(S_{i}\right) \cup\{\emptyset\}$ be a function such that $\psi_{2}\left(S_{2}\right)=S_{2}$ and $\phi=\psi_{1} \times \psi_{2}$. Then $A_{1} \times S_{2}$ is a $\phi$-quasi-prime ideal of $S_{1} \times S_{2}$ if and only if $A_{1}$ is a $\psi_{1}$-quasi-prime ideal of $S_{1}$.

Proof First assume that $A_{1} \times S_{2}$ is a $\phi$-quasi-prime ideal of $S_{1} \times S_{2}$. The proof is trivial and hence omitted.

Conversely, assume that $A_{1}$ is a $\psi_{1}$-quasi-prime ideal of $S_{1}$. Let $\left(a_{1}, b_{1}\right)$ and $\left(a_{2}, b_{2}\right)$ be any elements of $S_{1} \times S_{2}$ be such that

$$
\begin{aligned}
\left(a_{1} a_{2}, b_{1} b_{2}\right) & =\left(a_{1}, b_{1}\right)\left(a_{2}, b_{2}\right) \in A_{1} \times S_{2}-\phi\left(A_{1} \times S_{2}\right) \\
& =A_{1} \times S_{2}-\left(\psi_{1} \times \psi_{2}\right)\left(A_{1} \times S_{2}\right) \\
& =A_{1} \times S_{2}-\psi_{1}\left(A_{1}\right) \times S_{2} .
\end{aligned}
$$

Obviously, $a_{1} a_{2} \in A_{1}-\psi_{1}\left(A_{1}\right)$. By assumption, $a_{1} \in A_{1}$ or $a_{2} \in A_{1}$. Consequently, $A_{1}$ is $\psi_{1}$-quasi-prime ideal of $S_{1}$.

From Theorem 21 we can easily obtain the following theorem.

Theorem 22 Let $S_{1}$ and $S_{2}$ be two ordered $\mathcal{L} \mathcal{A}$-semigroups with left identities and let $\psi_{i}$ : $\mathcal{I}\left(S_{i}\right) \rightarrow \mathcal{I}\left(S_{i}\right) \cup\{\emptyset\}$ be a function such that $\psi_{1}\left(S_{1}\right)=S_{1}$ and $\phi=\psi_{1} \times \psi_{2}$. Then $S_{1} \times A_{2}$ is a $\phi$-quasi-prime ideal of $S_{1} \times S_{2}$ if and only if $A_{2}$ is a $\psi_{2}$-quasi-prime ideal of $S_{2}$.

From Theorems 21,22 we can easily obtain the following theorem.

Theorem 23 Let $S_{i}$ be an ordered $\mathcal{L} \mathcal{A}$-semigroup with left identity and let $\psi_{i}: \mathcal{I}\left(S_{i}\right) \rightarrow$ $\mathcal{I}\left(S_{i}\right) \cup\{\varnothing\}$ be a function such that $\psi_{j}\left(S_{j}\right)=S_{j}$ and $\phi=\psi_{1} \times \ldots \times \psi_{k}$. Then $S_{1} \times S_{2} \times$ $\ldots \times S_{i-1} \times A_{i} \times S_{i+1} \times \ldots \times S_{k}$ is a $\phi$-quasi-prime ideal of $S_{1} \times \ldots \times S_{k}$ if and only if $A_{i}$ is a $\psi_{i}$-quasi-prime ideal of $S_{i}$. 
Next, we show that if $A_{1} \times A_{2}$ is a $\phi$-quasi-prime ideal of an ordered $\mathcal{L} \mathcal{A}$-semigroup $S_{1} \times S_{2}$, then $A_{i}$ is a $\psi_{1}$-quasi-prime ideal of $S_{i}$ for all $i=1,2$.

Theorem 24 Let $A_{1}$ and $A_{2}$ be any proper left ideals of ordered $\mathcal{L} \mathcal{A}$-semigroups with left identities $S_{1}$ and $S_{2}$, respectively and let $\psi_{i}: \mathcal{I}\left(S_{i}\right) \rightarrow \mathcal{I}\left(S_{i}\right) \cup\{\emptyset\}$ be a function such that $\phi=\psi_{1} \times \psi_{2}$. Then the following properties hold:

1. If $A_{1} \times A_{2}$ is a $\phi$-quasi-prime ideal of $S_{1} \times S_{2}$ such that $A_{2} \neq \psi_{2}\left(A_{2}\right)$, then $A_{1}$ is a $\psi_{1}$-quasi-prime ideal of $S_{1}$.

2. If $A_{1} \times A_{2}$ is a $\phi$-quasi-prime ideal of $S_{1} \times S_{2}$ such that $A_{1} \neq \psi_{1}\left(A_{1}\right)$, then $A_{2}$ is a $\psi_{2}$-quasi-prime ideal of $S_{2}$.

Proof 1. Let $a_{1}$ and $a_{2}$ be any elements of $S_{1}$ be such that $a_{1} a_{2} \in A_{1}-\psi\left(A_{1}\right)$. If $A_{2} \neq$ $\psi_{2}\left(A_{2}\right)$, then there exists an element $c$ of $S_{2}$ such that $c \notin \psi_{2}\left(A_{2}\right)$. This implies that, $\left(a_{1}, e\right)\left(a_{2}, c\right)=\left(a_{1} a_{2}, c\right) \in A_{1} \times A_{2}-\psi_{1}\left(A_{1}\right) \times \psi_{2}\left(A_{2}\right)=A_{1} \times A_{2}-\phi\left(A_{1} \times A_{2}\right)$. Since $A_{1} \times A_{2}$ is a $\phi$-quasi-prime ideal of $S_{1} \times S_{2}$, we have $\left(a_{1}, e\right) \in A_{1} \times S_{2}$ or $\left(a_{2}, c\right) \in A_{1} \times S_{2}$. Therefore, $a_{1} \in A_{1}$ or $a_{2} \in A_{1}$ and hence $A_{1}$ is a $\psi_{1}$-quasi-prime ideal of $S_{1}$.

2. This follows from part 1 .

From Theorem 24 we can easily obtain the following theorem.

Theorem 25 Let $A_{i}$ be a proper left ideal of an ordered $\mathcal{L} \mathcal{A}$-semigroup $S_{i}$ with left identity and let $\psi_{i}: \mathcal{I}\left(S_{i}\right) \rightarrow \mathcal{I}\left(S_{i}\right) \cup\{\emptyset\}$ be a function such that $\phi=\psi_{1} \times \psi_{2} \times \ldots \times \psi_{k}$. If $A_{1} \times A_{2} \times \ldots \times A_{k}$ is a $\phi$-quasi-prime ideal of $S_{1} \times S_{2} \times \ldots \times S_{k}$ such that $A_{j} \neq \psi_{j}\left(A_{j}\right)$, then $A_{i}$ is a $\psi_{i}$-quasi-prime ideal of $S_{i}$.

The next theorem gives conditions for a $\phi$-quasi-prime ideal to be quasi-prime ideal in an ordered $\mathcal{L} \mathcal{A}$-semigroup.

Theorem 26 Let $S_{i}$ be an ordered $\mathcal{L} \mathcal{A}$-semigroup with left identity and left zero and let $\psi_{i}: \mathcal{I}\left(S_{i}\right) \rightarrow \mathcal{I}\left(S_{i}\right) \cup\{\emptyset\}$ be a function such that $\psi_{i}\left(S_{i}\right) \neq S_{i}$ and $\phi=\psi_{1} \times \psi_{2}$. If $A$ is a $\phi$-quasi-prime ideal of $S_{1} \times S_{2}$, then $A=\phi(A)$ or $A$ is a quasi-prime ideal of $S_{1} \times S_{2}$.

Proof Suppose that $A$ is a $\phi$-quasi-prime ideal of $S_{1} \times S_{2}$ that is not a quasi-prime ideal of $S_{1} \times S_{2}$. To show that $A \neq \phi(A)$. First assume, $A_{1} \times A_{2}=A \neq \phi(A)=\phi\left(A_{1} \times A_{2}\right)=$ $\psi_{1}\left(A_{1}\right) \times \psi_{2}\left(A_{2}\right)$. Then there exists an element $i$ of $\{1,2\}$ such that $A_{i} \neq \psi_{i}\left(A_{i}\right)$. We may assume that $A_{1} \neq \psi_{1}\left(A_{1}\right)$, there exists an element $c_{1}$ of $A_{1}$ such that $c_{1} \notin \psi_{1}\left(A_{1}\right)$. We will to show that $A_{2}=S_{2}$. Next, assume, $A_{2} \neq S_{2}$, it follows that there exists an element $c_{2}$ of $S_{2}$ such that $c_{2} \notin A_{2}$. In fact, since $\left(e, c_{2}\right)\left(c_{1}, e\right)=\left(c_{1}, c_{2} e\right) \notin \psi_{1}\left(A_{1}\right) \times \psi_{2}\left(A_{2}\right)=\phi(A)$, we have $\left(e, c_{2}\right)\left(c_{1}, e\right) \in A-\phi(A)$. Thus, $\left.\left(e, c_{2}\right)\right) \in A$ or $\left(c_{1}, e\right) \in A$. Obviously, $c_{2} \in A_{2}$, which is a contradiction. Therefore, $A=A_{1} \times S_{2}$, which means that $(0, e) \in A$. By Theorem 14 ,

$$
\begin{aligned}
(0, e) & =(0, e)^{2} \in A^{2} \\
& =\phi_{2}(A) \\
& \subseteq \phi(A) \\
& =\psi_{1}\left(A_{1}\right) \times \psi_{2}\left(A_{2}\right),
\end{aligned}
$$

which is a contradiction. Hence, $A=\phi(A)$.

From Theorem 26 we can easily obtain the following theorem. 
Theorem 27 Let $S_{i}$ be an ordered $\mathcal{L} \mathcal{A}$-semigroup with left identity and left zero and let $\psi_{i}: \mathcal{I}\left(S_{i}\right) \rightarrow \mathcal{I}\left(S_{i}\right) \cup\{\emptyset\}$ be a function such that $\psi_{i}\left(S_{i}\right) \neq S_{i}$ and $\phi=\psi_{1} \times \psi_{2} \times \ldots \times \psi_{k \geq 2}$. If $A$ is a $\phi$-quasi-prime ideal of $S_{1} \times S_{2} \times \ldots \times S_{k}$, then $A=\phi(A)$ or $A$ is a quasi-prime ideal of $S_{1} \times S_{2} \times \ldots \times S_{k}$.

The above theorem shows the relationship between quasi-prime ideals and $\phi$-quasi-prime ideals in an ordered $\mathcal{L} \mathcal{A}$-semigroup $S_{1} \times S_{2}$. From the above theorem, we have the following theorem.

Theorem 28 Let $S_{i}$ be an ordered $\mathcal{L} \mathcal{A}$-semigroup with left identity and left zero and let $\psi_{i}: \mathcal{I}\left(S_{i}\right) \rightarrow \mathcal{I}\left(S_{i}\right) \cup\{\emptyset\}$ be a function such that $\psi_{i}\left(S_{i}\right) \neq S_{i}, \phi=\psi_{1} \times \psi_{2}$ and $A \neq \phi(A)$. Then $A$ is a $\phi$-quasi-prime ideal of $S_{1} \times S_{2}$ if and only if $A$ is a quasi-prime ideal of $S_{1} \times S_{2}$.

Proof This follows from Theorem 26.

From Theorem 27 we can easily obtain the following theorem.

Theorem 29 Let $S_{i}$ be an ordered $\mathcal{L} \mathcal{A}$-semigroup with left identity and left zero and let $\psi_{i}: \mathcal{I}\left(S_{i}\right) \rightarrow \mathcal{I}\left(S_{i}\right) \cup\{\emptyset\}$ be a function such that $\psi_{i}\left(S_{i}\right) \neq S_{i}, \phi=\psi_{1} \times \psi_{2} \times \ldots \times \psi_{k \geq 2}$ and $A \neq \phi(A)$. Then $A$ is a $\phi$-quasi-prime ideal of $S_{1} \times S_{2} \times \ldots \times S_{k}$ if and only if $A$ is a quasi-prime ideal of $S_{1} \times S_{2} \times \ldots \times S_{k}$.

As a simple consequence of Theorem 6, we give the following result.

Lemma 1 Let $\phi: \mathcal{I}(S) \rightarrow \mathcal{I}(S) \cup\{\emptyset\}$

be a function and let $A$ be a left ideal of an ordered $\mathcal{L} \mathcal{A}$-semigroup $S$ that is not a quasiprime ideal. If $A$ is a $\phi$-quasi-prime ideal of $S$ such that $\phi \leq \phi_{2}$, then $A^{2}=A^{n+1}$.

The next theorem gives conditions for a $\phi$-quasi-prime ideal to be $\omega$-quasi-prime ideal in a commutative semigroup.

Theorem 30 Let $\phi: \mathcal{I}(S) \rightarrow \mathcal{I}(S) \cup\{\emptyset\}$ be a function where $\phi \leq \phi_{n+1}$. Then $A$ is a $\phi$-quasi-prime ideal of $S$ if and only if $A$ is an $\omega$-quasi-prime ideal of $S$.

Proof First assume that $A$ is a $\phi$-quasi-prime ideal of $S$. If $A$ is a quasi-prime ideal of $S$, then it is $\omega$-quasi-prime ideal. Now assume that $A$ is not a quasi-prime ideal of $S$. Then by Lemma $1, A^{2}=A^{n+1}$. By assumption, $A$ is a $\phi$-quasi-prime ideal of $S$ and $\phi \leq \phi_{n+1}$, which implies that $A$ is a $\phi_{n+1}$-quasi-prime ideal of $S$. On the other hand, $\phi_{\omega}(A)=A^{n+1}=\phi_{n+1}(A)$. Therefore $A$ is an $\omega$-quasi-prime ideal of $S$.

Conversely, assume that $A$ is a $\phi$-quasi-prime ideal of $S$. The proof is trivial and hence omitted.

\section{Conclusion}

In study the structure of ordered $\mathcal{L} \mathcal{A}$-semigroups, we notice that the quasi-prime ideals with special properties always play an important role. The purposes of this paper are to introduce generalizations of quasi-prime ideals to the context of $\phi$-quasi-prime ideals. Some characterizations of quasi-prime and $\phi$-quasi-prime ideals are obtained. Moreover, we investigate relationships between weakly quasi-prime, almost quasi-prime, $\omega$-quasi-prime, $m$-quasiprime and $\phi$-quasi-prime ideals of ordered $\mathcal{L} \mathcal{A}$-semigroups. Finally, we obtain necessary and sufficient conditions of $\phi$-quasi-prime ideal in order to be a quasi-prime ideal. 
Open Access This article is licensed under a Creative Commons Attribution 4.0 International License, which permits use, sharing, adaptation, distribution and reproduction in any medium or format, as long as you give appropriate credit to the original author(s) and the source, provide a link to the Creative Commons licence, and indicate if changes were made. The images or other third party material in this article are included in the article's Creative Commons licence, unless indicated otherwise in a credit line to the material. If material is not included in the article's Creative Commons licence and your intended use is not permitted by statutory regulation or exceeds the permitted use, you will need to obtain permission directly from the copyright holder. To view a copy of this licence, visit http://creativecommons.org/licenses/by/4.0/.

\section{References}

1. Abbasi, M.Y., Basar, A.: On generalizations of ideals in LA- $\Gamma$-semigroups. Southeast Asian Bull. Math. 39, 1-12 (2015)

2. Abbasi, M.Y., Khan, S.A., Talee, A.F., Khan, A.: Soft interior-hyperideals in left regular LAsemihypergroups. Kragujevac J. Math. 44(2), 217-236 (2020)

3. Abdullah, S., Aslam, S., Amin, N.: LA-semigroups characterized by the properties of interval valued $(\alpha, \beta)$-fuzzy ideals. J. Appl. Math. Informatics 32(3-4), 405-426 (2014)

4. Ali, A., Shi, F.G., Khan, M.: Characterizations of ordered AG-groupoids in terms of soft sets. Italian J. Pure Appl. Math. 35, 415-432 (2015)

5. Amjad, V., Hila, K., Yousafzai, F.: Generalized hyperideals in locally associative left almost semihypergroups. N. Y. J. Math. 20, 1063-1076 (2014)

6. Amjid, V., Yousafzai, F., Hila, K.: A study of ordered AG-groupoids in terms of semilattices via smallest (fuzzy) ideals. Adv. Fuzzy Syst. 2018, 8 (2018)

7. Amjad, V., Yousafzai, F., Iampan, A.: On generalized fuzzy ideals of ordered LA-semigroups. Boletin de Mat. 22(1), 1-19 (2015)

8. Anderson, D.D., Bataineh, M.: Generalizations of prime ideals. Commun. Algebra 36(2), 686-696 (2015)

9. Davvaz, B., Khan, M., Anis, S., Haq, S.: Generalized fuzzy quasi-ideals of an intraregular AbelGrassmann's groupoid. J. Appl. Math. 2012, 16 (2012)

10. Dudek, W.A., Gigon, R.S.: Completely inverse AG**-groupoids. Semigroup Forum 87, 201-229 (2013)

11. Faisal, A.K., Davvaz, B.: On fully regular AG-groupoids. Afr. Mat. 25(2), 449-459 (2014)

12. Faisal, N.Y., Ghareeb, A.: Left regular AG-groupoids in terms of fuzzy interior ideals. Afr. Mat. 24(4), 577-587 (2013)

13. Faisal, Y.N., Hila, K.: On fuzzy $(2,2)$-regular ordered $\Gamma$-AG** -groupoids. UPB Sci. Bull. Ser. A 74(2), 87-104 (2012)

14. Faisal, N.Y., Saeid, A.B.: Some results in bipolar-valued fuzzy ordered AG-groupoids. Discuss. Math. General Algebra Appl. 32, 55-76 (2012)

15. Feng, F., Khan, M., Fotea, V.L., Anis, V., Ajaib, N.: Fuzzy soft set approach to ideal theory of regular AG-groupoids. An. St. Univ. Ovidius Constanta 24(1), 263-288 (2016)

16. Iqbal, M., Ahmad, I.: Ideals in CA-AG-groupoids. Indian J. Pure Appl. Math. 49(2), 265-284 (2018)

17. Khan, M., Anis, S.: On semilattice decomposition of an Abel-Grassmann's Groupoid. Acta Math. Sin. Engl. Ser. 28(7), 1461-1468 (2012)

18. Khan, A., Faisal, W.K., Yaqoob, N.: Ordered LA-semigroups in terms of interval valued fuzzy ideals. J. Adv. Res. Pure Math. 5(1), 100-117 (2013)

19. Khan, A., Farooq, M., Izhar, M., Davvaz, B.: Fuzzy hyperideals of left almost semihypergroups. Int. J. Anal. Appl. 15(2), 155-171 (2018)

20. Khan, M., Jun, Y.B., Yousafzai, F.: Fuzzy ideals in right regular LA-semigroups. Hacettepe J. Math. Stat. 44(3), 569-586 (2015)

21. Khan, F.M., Khan, H.U., Mukhtar, S., Khan, A., Sarmin, N.H.: Some innovative types of fuzzy ideals in AG-groupoids. J. Intell. Syst. 1-19, 5 (2013)

22. Khan, M., Mushtaq, Q., Anis, S.: A note on Abel-Grassmann's groupoids. Res. J. Appl. Sci. Eng. Technol. 7(9), 1705-1709 (2014)

23. Khan, M., Shum, K.P., Iqbal, M.F.: On minimal ideals of AG**-groupoids. Southeast Asian Bull. Math. 38, 389-399 (2014)

24. Khan, W., Yousafzai, F., Khan, A.: On ordered Abel-Grassmann's groupoids. Probl. Phys. Math. Technics 2(23), 40-47 (2015)

25. Khan, W., Yousafzai, F., Khan, M.: On generalized ideals of left almost semigroups. Eur. J. Pure Appl. Math. 9(3), 277-291 (2016)

26. Mushtaq, Q.: Zeroids and idempoids in AG-groupoids. Quasigroups Relat. Syst. 11, 79-84 (2004) 
27. Mushtaq, Q., Khan, M.: Decomposition of AG*-groupoids. Quasigroups Relat. Syst. 15, 303-308 (2007)

28. Mushtaq, Q., Khan, M.: Ideals in AG-band and AG*-groupoid. Quasigroups Relat. Syst. 14, 207-215 (2006)

29. Rehman, N., Park, C., Shah, S.I.A., Ali, A.: On generalized roughness in LA-semigroups. Mathematics 6(7), 1-8 (2018)

30. Rehman, N., Shah, N., Ali, M.I., Ali, A.: Generalised roughness in $(\in, \in \vee q)$-fuzzy substructures of LA-semigroups. J. Natn. Sci. Found. Sri Lanka 46(3), 465-473 (2018)

31. Sezer, A.S.: A new approach to LA-semigroup theory via the soft sets. J. Intell. Fuzzy Syst. 26(5), 2483-2495 (2014)

32. Sezgin, A.: A new view on AG-groupoid theory via soft sets for uncertainty modeling. Filomat 32(8), 2995-3030 (2018)

33. Shah, T., Kausar, N.: Characterizations of non-associative ordered semigroups by their fuzzy bi-ideals. Theor. Comput. Sci. 529, 96-110 (2014)

34. Shah, T., Rehman, I.: Decomposition of locally associative $\Gamma$-AG-groupoids. Novi Sad. J. Math. 43(1), 1-8 (2013)

35. Shah, T., Rehman, I., Chinram, R.: On M-systems in ordered AG-groupoids. Far East J. Math. Sci. 47(1), 13-21 (2010)

36. Shah, T., Rehman, I., Khan, A.: Fuzzy $\Gamma$-ideals in $\Gamma$-AG-groupoids. Hacettepe J. Math. Stat. 43(4), 625-634 (2014)

37. Stevanovic, N., Proti, P.V.: Composition of Abel-Grassmann's 3-bands. Novi Sad J. Math. 34(2), 175-182 (2004)

38. Yaqoob, N., Chinram, R., Ghareeb, A., Aslam, M.: Left almost semigroups characterized by their interval valued fuzzy ideals. Afr. Mat. 24(2), 231-245 (2013)

39. Yaqoob, N., Corsini, P., Yousafzai, F.: On intra-regular left almost semihypergroups with pure left identity. J. Math. 2013, 10 (2013)

40. Yiarayong, P.: On semiprime and quasi semiprime ideals in AG-groupoids. KKU Res. J. 18(6), 893-896 (2013)

41. Yiarayong, P.: On product of fuzzy semiprime ideals in $\Gamma$-LA-semigroups. GU J. Sci. 29(2), 491-502 (2016)

42. Yiarayong, P.: On prime and semiprime ideals in ordered AG-groupoids. Iran. J. Math. Sci. Informatics 12(1), 1-11 (2017)

43. Younas, I., Mushtaq, Q.: On left permutable inverse LA-semigroups. Maejo Int. J. Sci. Technol. 13(01), 10-18 (2019)

44. Yousafzai, F., Asif, T., Khan, A., Davvaz, B.: A study of non-associative ordered semigroups in terms of semilattices via smallest (double-framed soft) ideals. Int. J. Anal. Appl. 16(4), 484-502 (2018)

45. Yousafzai, F., Iampan, A., Tang, J.: Study on smallest (fuzzy) ideals of LA-semigroups. Thai J. Math. 16(2), 549-561 (2018)

46. Yousafzai, F., Khan, A., Amjad, V., Zeb, A.: On fuzzy fully regular ordered AG-groupoids. J. Intell. Fuzzy Syst. 26, 2973-2982 (2014)

47. Yousafzai, F., Khan, A., Khan, W., Aziz, T.: $\left(\in, \in \vee q_{k}\right)$-fuzzy ideals in left regular ordered LAsemigroups. Afr. Mat. 35(4), 583-606 (2013)

48. Yousafzai, F., Khan, W., Khan, A., Hila, K.: I-V fuzzy set theory applied on po-LA-semigroups. Afr. Mat. 27(1-2), 23-35 (2016)

49. Yousafzai, F., Khan, A., Iampan, A.: On $(m, n)$-ideals of an ordered Abel-Grassmann groupoid. Korean J. Math. 23(3), 357-370 (2015)

50. Yousafzai, F., Yaqoob, N., Haq, S., Manzoor, R.: A note on intuitionistic fuzzy $\Gamma$-LA-semigroups. World Appl. Sci. J. 19(12), 1710-1720 (2012)

51. Yousafzai, F., Yaqoob, N., Zeb, A.: On generalized fuzzy ideals of ordered AG-groupoids. Int. J. Mach. Learn. Cyber. 7(6), 995-1004 (2016)

Publisher's Note Springer Nature remains neutral with regard to jurisdictional claims in published maps and institutional affiliations. 